\title{
Investigation of Three-Phase Thyristor Converters under Generalized Impedance Unbalance
}

\author{
Manuel Weiland, Gerhard Herold \\ Institute of Electrical Power Systems \\ University of Erlangen-Nuremberg \\ Cauerstraße 4 - Haus 1, 91058 Erlangen, Germany \\ Phone/Fax number: +49 9131 85295-27/41, e-mail: weiland@eev.eei.uni-erlangen.de
}

\begin{abstract}
Line-commutated current converters operated in electrical power systems will work asymmetrically, if the system is not balanced. This asymmetrical operation causes non-characteristic harmonics in the currents and therefore a higher distortion level. As a high distortion level is always disadvantageous, it is worthwhile to find appropriate methods to lower the distortion level by reducing the amplitudes of these non-characteristic harmonics.

In this paper, three-phase thyristor converters operated in systems with unbalanced AC-line short-circuit impedances are investigated. To achieve a competent and well-grounded understanding of the problem a suitable method of calculation has been developed. This method firstly offers the opportunity to calculate the operation of line-commutated current converters in an unbalanced three-phase system. Furthermore it offers the opportunity to optimize the operation of the unbalanced system. This optimization is done by balancing the systems operation and reducing the amplitudes of the non-characteristic harmonics using asymmetrical firing angles.
\end{abstract}

\section{Key words}

Power converter, unbalance, converter control, distortion, optimization

\section{Introduction}

Electrical three-phase power systems are never perfectly balanced. These unbalances can be results of an unbalanced mains voltage, unbalanced loads or unbalanced AC-line short-circuit impedances. The latter case with unbalanced short-circuit impedances is investigated in this paper. An unbalance of this kind can be caused by faulty transmission lines or overhead transmission lines without transposition for example.

Current converters operated in unbalanced electrical systems will generally work asymmetrically. This is also valid for similar electrical power equipment, like FACTS, HVDC converters and current limiters based on power converters, when this equipment is operated in an unbalanced electrical system. In all of these cases this asymmetrical operation firstly introduces the appearance of non-characteristic harmonics. These non-characteristic harmonics lead to a distortion level, higher than the distortion occurring in case of a balanced operation of the system [1, 2]. As a high distortion level is always disadvantageous, both for the converter and the power system, a method to keep the distortion as low as possible is desirable.

For a given system the impedance unbalance is fixed and not changeable. So the reduction of the distortion level can only be done by reducing the amplitudes of the non-characteristic harmonics. With controllable current converters, such as thyristor converters, this reduction of the distortion level can be realized by balancing the current converter's operation using a control strategy with unsymmetrical firing angles [3, 4]. This makes the operation of a current converter in an unbalanced electrical power system more balanced and reduces the THD.

The aim is now to calculate optimal firing angles for a given system with a given impedance unbalance. Due to the unbalance, these optimal firing angels will be asymmetrical. This means for example for a six-pulse thyristor bridge in asymmetrical operation the spacing between two optimal firing impulses is not $60^{\circ}$ like in normal symmetrical operation.

This possibility with unsymmetrical firing angels has already been shown for forced-commutated converters both with an unbalanced mains voltage and with unbalanced AC-line impedances $[5,6]$ and for line-commutated power converters with an unbalanced mains voltage [7, 8]. A first approach has been shown for line-commutated converters with a simplified unbalance of the AC-lines short-circuit impedance [9]. In this work, a generalized approach is presented, wherein the simplifications of [9] do not have to be complied with. Hence the unbalance of the AC-lines short-circuit impedance can be of any kind and practical problems can be investigated more realistically.

In calculating the operation of current converters it is common to assume all impedances to be lossless and the 
direct current to be ideal smooth without any ripple. These simplifications do not have to be applied here, as an approach with differential equations is used in this paper. Because of the calculation with differential equations no requirements to the AC-line and DC impedances have to be complied with, except of linearity. In particular, this means that the AC-line impedance does not have to be negligible and may have a resistive part. The DC-network may have a non-infinite reactance, which corresponds to a ripple-afflicted direct current. Capacitive elements can be included both on the AC and DC side of the converter, which introduces the possibility to take filter into account.

In the following explanations we consider an electrical three-phase system with an unbalanced AC-lines short-circuit impedance. This unbalance is given and fixed and therefore not changeable. In this system a current converter is operated. From this current converter we know the DC-voltage and the desired operation point (e.g. a needed value of the direct current to charge the DC voltage source). For this converter system it will be shown how to derive optimal firing angles. These optimal firing angles will simultaneously fulfil the conditions of the desired operating point and minimize the distortion caused by non-characteristic harmonics.

\section{The Investigated System}

Fig. 1 shows the schematic diagram of the analysed system. It consists of a six-pulse line-commutated current converter with an impedance and a DC voltage source at its DC terminals.

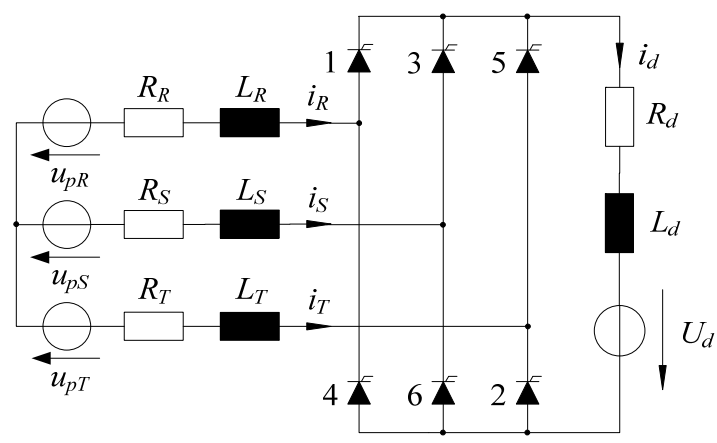

Fig. 1. Schematic diagram of the analysed system

The converter is fed by a symmetrical sinusoidal three-phase mains voltage $u_{p R, S, T}$. The AC-line short-circuit impedances are of ohmic-inductive type and are unbalanced

$$
\begin{aligned}
& R_{R} \neq R_{S} \neq R_{T}, \\
& L_{R} \neq L_{S} \neq L_{T} .
\end{aligned}
$$

The following demonstration will show a rectifier's operation of the current converter, for example charging the battery of an EV or feeding a DC railroad system. Though, the presented method is applicable to an inverter's operation, too. This offers the opportunity to take FACTS and HVDC systems into account. In case of an inverter's operation of the current converter only the DC voltage source $U_{d}$ has to be included in the calculations with its inversed sign.

\section{Calculation with Space-Phasors}

For easier analysis, the system is transformed into the image range of space-phasors. This transformation is defined for any three-phase system $v_{R}(\omega t), v_{S}(\omega t), v_{T}(\omega t)$

$$
\underline{v}(\omega t)=\frac{2}{3}\left(v_{R}(\omega t)+\underline{a} v_{S}(\omega t)+\underline{a}^{2} v_{T}(\omega t)\right)
$$

with $\underline{a}=\mathrm{e}^{\mathrm{j} 2 \pi / 3}$. Eq. (3) shows, the space-phasor is by definition a complex time-characteristic with a real part $v_{\alpha}(\omega t)$ and an imaginary part $v_{\beta}(\omega t)$. The three real time-characteristics $v_{R, S, T}(\omega t)$ are mapped into this one complex time-characteristic $\underline{v}(\omega t)$. A zero component can be omitted here, because in the analysed system the sum of all currents is always zero.

As the system will be analyzed with differential equations and to achieve manageable solution statements of the transient parts of the differential equations, a time reference is useful. This can be done via a phase angle $\gamma$, which is very similar to the well-known delay angle in common converter theory. In our case $\gamma$ is the delay between the maximum of the line-voltage $u_{p R}$ and the firing of thyristor 3 at $\omega t=0$. With this definition the effective mains voltages are

$$
u_{p R, S, T}(\omega t)=\hat{U}_{p} \cdot \cos \left(\omega t+\gamma+\varphi_{R, S, T}\right)
$$

with $\varphi_{R}=0, \varphi_{S}=-2 \pi / 3, \varphi_{T}=-4 \pi / 3$.

Eq. (4) inserted in eq. (3) leads to a circular shaped space-phasor of the mains voltage

$$
\underline{u}_{p}(\omega t)=\hat{U}_{p} \cdot \mathrm{e}^{\mathrm{j}(\omega t+\gamma)}=u_{p \alpha}(\omega t)+\mathrm{j} u_{p \beta}(\omega t) .
$$

As eq. (4) shows, the system is excited with sinusoidal voltages. Sinusoidal functions are symmetrical by $\pi$. So the excitation of the converter system is symmetrical by $\pi$ and all currents and voltages of the converter system are forced to be at least symmetrical by $\pi$. This leads to the following condition for every time characteristic of the original three-phase system

$$
v_{R, S, T}(\omega t+(2 k+1) \pi)=-v_{R, S, T}(\omega t+2 k \pi) .
$$

Herein is $k \in \mathbb{N}_{0}$ and $v_{R, S, T}(\omega t)$ stands for the time characteristics of currents or voltages. With eq. (6) and the asymmetrical short-circuit impedance it is possible to show all time-characteristics are exactly symmetrical by $\pi$. This means for example the direct current of the six-pulse converter will not be periodical with $2 \pi / 6=60^{\circ}$ as normal in symmetrical operation. Instead, because of the asymmetrical short-circuit impedances, the direct current will be periodical with $\pi=180^{\circ}$.

As a full $50 \mathrm{~Hz}$-period corresponds to an angle of $2 \pi=360^{\circ}$ two of these $180^{\circ} \mathrm{DC}$-periods occur during one $50 \mathrm{~Hz}$-period. So this periodicity with $\pi$ is called two-pulse type operation mode. This two-pulse type operation mode is the asymmetrical operation of the converter investigated in this work.

During one two-pulse period of the duration $\pi$ there are six conducting states of the current converter. Table I shows the active thyristors during these states. 
Table I. - Conducting states of one two-pulse period

\begin{tabular}{|c|c|}
\hline Conducting state & Active thyristors \\
\hline $\mathbf{z}_{1}$ & 1,2 , and 3 \\
\hline $\mathbf{z}_{2}$ & 2 and 3 \\
\hline $\mathbf{z}_{3}$ & 2,3 and 4 \\
\hline $\mathbf{z}_{4}$ & 3 and 4 \\
\hline $\mathbf{z}_{5}$ & 3,4 and 5 \\
\hline $\mathbf{z}_{6}$ & 4 and 5 \\
\hline
\end{tabular}

Fig. 2 shows the conducting states and the time intervals in which they are valid. Herein for example $\beta_{1}$ is the angle in time thyristor 1 stops conducting, which corresponds to the end of commutation. In asymmetrical operation the six states will generally differ in their duration.

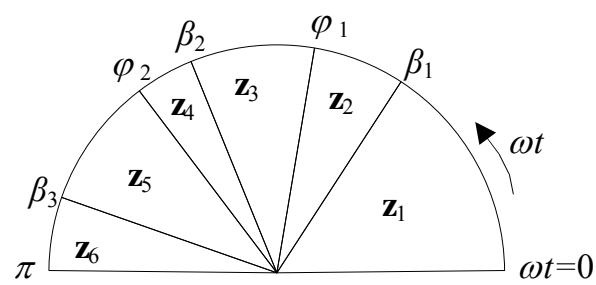

Fig. 2. Conducting states and angles in time during one two-pulse period

To calculate the asymmetrical operation in the range of space-phasors, the system's space-phasor description has to be derived. This has to be done separately for every conducting state of table I. For example, in conducting state $\mathbf{z}_{1}$ the currents $i_{R}$ and $i_{S}$ are positive with respect to fig. 1, while $i_{T}$ is negative. After inserting this in the space-phasor equation (3) and considering the two mesh equations of fig. 1 in state $\mathbf{z}_{1}$, one gets the space-phasor network for state $\mathbf{z}_{1}$. It is shown in fig. 3 .

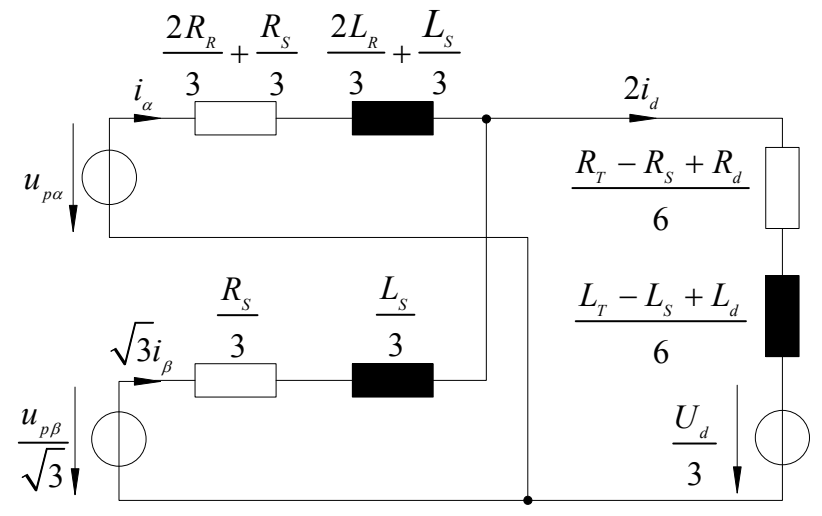

Fig. 3. Space-phasor network for conducting state $\mathbf{z}_{1}$

The space-phasor networks for the other five conducting states are different from each other, but are derived similarly to fig. 3 .

\section{Description in the State-Space}

The two meshes in fig. 3 lead to two differential equations for state $\mathbf{z}_{1}$

$$
\frac{\mathrm{d}}{\mathrm{d} \omega t} i_{\alpha}(\omega t)=\mathrm{f}_{1 \alpha}\left(i_{\alpha, \beta}(\omega t), u_{p \alpha, \beta}(\omega t), U_{d}, R_{R, S, T, d}, L_{R, S, T, d}\right),(7)
$$

$$
\frac{\mathrm{d}}{\mathrm{d} \omega t} i_{\beta}(\omega t)=\mathrm{f}_{1 \beta}\left(i_{\alpha, \beta}(\omega t), u_{p \alpha, \beta}(\omega t), U_{d}, R_{R, S, T, d}, L_{R, S, T, d}\right) .
$$

The other two conducting states with three conducting thyristors ( $\mathbf{z}_{3}$ and $\mathbf{z}_{5}$ ) lead to similar equations. In the case of two conducting thyristors (states $\mathbf{z}_{2}, \mathbf{z}_{4}$ and $\mathbf{z}_{6}$ ) the space-phasor network does not contain a node. So, for all conducting states with only two active thyristors only one equation in the form of (8) is needed. This leads to a description with space vectors for all states with three active thyristors

$$
\mathbf{z}_{1,3,5}=\left[\begin{array}{c}
i_{1,3,5 \alpha}(\omega t) \\
i_{1,3,5 \beta}(\omega t)
\end{array}\right]=\left[\begin{array}{c}
z_{1,3,5 \alpha} \\
z_{1,3,5 \beta}
\end{array}\right],
$$

while all states with two active thyristors contain only one state variable

$$
\mathbf{z}_{2,4,6}=i_{2,4,6 \beta}(\omega t)=z_{2,4,6} .
$$

With eq. (7) to (10) for every conducting state of table I a state space description can be stated

$$
\frac{\mathrm{d}}{\mathrm{d} \omega t} \mathbf{z}_{1, \ldots, 6}=\mathbf{A}_{1, \ldots, 6} \cdot \mathbf{z}_{1, \ldots, 6}+\mathbf{B}_{p 1, \ldots, 6} \cdot\left[\begin{array}{c}
u_{p \alpha} \\
u_{p \beta}
\end{array}\right]+\mathbf{b}_{d 1, \ldots, 6} \cdot U_{d} .
$$

As eq. (11) is valid for all conducting states, altogether one gets six equations of this form. In the following the indices $1, \ldots, 6$ of the conducting states are omitted. Without further distinction the matrices $\mathbf{A}$ and $\mathbf{B}_{p}$ and the vector $\mathbf{b}_{d}$ are different for every conducting state.

To solve the problem of eq. (11) steady state (index $s$ ) and transient/homogeneous (index $h$ ) solution statements have to be set up in common ways. The steady-state AC solutions $\mathbf{z}_{S A C}$ are stated with complex AC analysis, the steady-state DC solutions $\mathbf{z}_{S D C}$ with Ohm's law.

$$
\begin{gathered}
\mathbf{z}_{s A C}=\operatorname{Re}\left\{-[\mathbf{A}-\mathrm{j} \mathbf{E}]^{-1} \cdot \mathbf{B}_{p} \cdot\left[\begin{array}{l}
\hat{U}_{p \alpha} \\
\hat{U}_{p \beta}
\end{array}\right] \cdot \mathrm{e}^{\mathrm{j} \omega t}\right\}, \\
\mathbf{z}_{s D C}=-\mathbf{A}^{-1} \mathbf{b}_{d} U_{d} .
\end{gathered}
$$

The transient solution statements are set up with the matrix exponential

$$
\mathbf{z}_{h}=\mathrm{e}^{\mathrm{A} \cdot\left(\omega t-\omega t_{0}\right)} \cdot \mathbf{c}=\mathbf{V}\left[\begin{array}{cc}
\mathrm{e}^{p_{1}\left(\omega t-\omega t_{0}\right)} & 0 \\
0 & \mathrm{e}^{p_{2}\left(\omega t-\omega t_{0}\right)}
\end{array}\right] \mathbf{V}^{-1} \cdot \mathbf{c} .(14
$$

Herein is $\mathbf{V}$ the eigenvector-matrix and $p$ are the eigenvalues of one conduction state. The vector c contains the transient solutions' initial values. $\mathbf{V}, p$ and $\mathbf{c}$ are different for every conducting state. The start angle $\omega t_{0}$ stands for the angle the according conducting state begins (see fig. 2). That is $\omega t_{0}=\varphi_{1}$ for $\mathbf{z}_{3}$ for example. Finally one gets the complete solution statement by summation of the separate solution statements

$$
\mathbf{z}=\mathbf{z}_{s A C}+\mathbf{z}_{s D C}+\mathbf{z}_{h}
$$

The summands of eq. (15) are different for every conducting state. 


\section{Solution with Continuity Constraints}

Now the solution statements for every conducting state are known, but there are still some unknowns: All conducting states with three active thyristors $\left(\mathbf{z}_{1}, \mathbf{z}_{3}\right.$ and $\mathbf{z}_{5}$ ) contain two initial values of the transient solution parts, all conducting states with two active thyristors $\left(\mathbf{z}_{2}\right.$, $\mathbf{z}_{4}$ and $\mathbf{z}_{6}$ ) contain only one. These nine initial values are unknown. Furthermore the commutation stop angles $\beta_{1}$, $\beta_{2}$ and $\beta_{3}$ are unknown. To determine these twelve unknowns the continuity of the state vectors (see fig. 2 and eq. (9) and (10)) is used. The state vectors' elements are currents and therefore have to be continuous at every change of the conducting state. This is shown here only for the first change of the conducting state at $\omega t=\beta_{1}$

$$
\left[\begin{array}{c}
z_{1 \alpha}\left(\omega t=\beta_{1}-0\right) \\
z_{1 \beta}\left(\omega t=\beta_{1}-0\right)
\end{array}\right]=\left[\begin{array}{c}
0 \\
z_{2}\left(\omega t=\beta_{1}+0\right)
\end{array}\right] .
$$

For the conducting state changes at $\varphi_{1}, \beta_{2}, \varphi_{2}$ and $\beta_{3}$ equations analogue to (16) can be stated. So additionally to the two equations of (16) one gets eight more continuity equations (two equations per conducting state change). As seen in fig. 2, the calculation begins with $\omega t=0$ and ends with $\omega t=\pi$. But because of eq. (6) the state vectors reiterate periodically by $\pi$ and these two last conducting state changes at $\omega t=0$ and $\omega t=\pi$ appear like only one conducting state change in the calculation

$$
\left[\begin{array}{c}
\sqrt{3} \cdot z_{6}(\omega t=\pi-0) \\
z_{6}(\omega t=\pi-0)
\end{array}\right]=\left[\begin{array}{c}
-z_{1 \alpha}(\omega t=0+0) \\
-z_{1 \beta}(\omega t=0+0)
\end{array}\right] .
$$

All in all these six conducting state changes result in twelve equations. In these twelve equations the elements of the state vectors are substituted with their solution statements of eq. (15). For example this substitution in the second line of eq. (16) results in

$$
\begin{aligned}
& z_{2 s A C}\left(\omega t=\beta_{1}\right)+z_{2 s D C}+z_{2 h}\left(\omega t=\beta_{1}\right) \\
& =z_{1 \beta s A C}\left(\omega t=\beta_{1}\right)+z_{1 \beta s D C}+z_{1 \beta h}\left(\omega t=\beta_{1}\right) .
\end{aligned}
$$

Now for the twelve unknowns twelve equations are stated and the problem is solvable [9].

\section{Degrees of Freedom}

The angles $\varphi_{1}$ and $\varphi_{2}$ in fig. 2 define the firing impulses of the thyristors 4 and 5 . In symmetrical operation these angels are always

$$
\varphi_{1}=60^{\circ}, \varphi_{2}=120^{\circ} \text {. }
$$

In asymmetrical operation these two firing angles are degrees of freedom and can be chosen out of a reasonable range. This can be for example

$$
\varphi_{1} \in\left[55^{\circ} ; 65^{\circ}\right], \varphi_{2} \in\left[115^{\circ} ; 125^{\circ}\right] .
$$

These two degrees of freedom are used to optimize the asymmetrical operation of the current converter, especially to reduce the THD.

Now, for a given electrical power system with a known impedance unbalance and a desired working point of the converter (e.g. a needed mean-value of the direct current), the problem is solvable for every pair of $\varphi_{1}$ and $\varphi_{2}$ of eq. (20). Every pair leads to the needed mean-value of the direct current. But besides that, every pair of $\varphi_{1}$ and $\varphi_{2}$ leads to different time-characteristics of the AC-line currents. So every working point, defined by a pair of $\varphi_{1}$ and $\varphi_{2}$, leads to a different quality of the converter operation. In the following example the THD will be used as quality criterion. It will be shown how the THD depends on $\varphi_{1}$ and $\varphi_{2}$ and how the degrees of freedom can be used to reduce the THD in asymmetrical operation.

\section{Calculation of an Example}

Considered is a current converter for a $750 \mathrm{~V}$ DC railroad system. The DC-power is $900 \mathrm{~kW}$, so we need an arithmetical mean value of the direct current of

$$
i_{d_{\text {mean }}}=1,2 \mathrm{kA} \text {. }
$$

The system is supplied by a $10 \mathrm{kV} / 690 \mathrm{~V}$ transformer with a rated power of $1600 \mathrm{kVA}$. The transformer is the major part of the system's short-circuit impedance. The system's overall short-circuit impedance is unbalanced because of AC-lines without transposition

$$
\begin{gathered}
R_{R}=R_{S}=R_{T}=2,8 \mathrm{~m} \Omega, \\
\omega L_{R}=\omega L_{S}=1,36 \cdot 18 \mathrm{~m} \Omega, \\
\omega L_{T}=18 \mathrm{~m} \Omega .
\end{gathered}
$$

On the DC-side the impedance is

$$
L_{d}=100 \mathrm{~m} \Omega, R_{d}=L_{d} / 20 .
$$

With these parameters the shown method of calculation can be executed within the range of eq. (20). Fig. 4 shows the THD of the AC-line currents within this range; one sees it is least distorted for

$$
\varphi_{1}=60^{\circ}, \varphi_{2}=120,8^{\circ} \text {. }
$$

The THD in this case is $31,42 \%$.

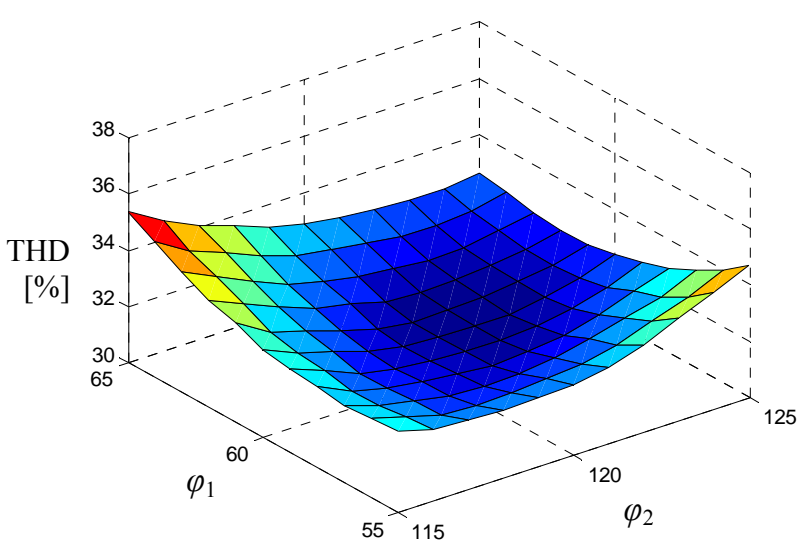

Fig. 4. THD of the AC-line currents

This result is compared to the classical equally-pulse-spaced (EPS) firing method [3], which always uses the symmetrical firing angles defined in eq. (19). Using EPS the THD is $31,64 \%$. As this is only a small difference we look at the time-characteristics of the 
currents: Fig. 5 shows the time-characteristics of the direct current for the above mentioned minimization of the THD and for the EPS firing method. Therein the bold graph is for optimized firing angles (eq. (26)) and the thin one is for EPS (eq. (19)). One sees, besides a minimization of the THD, the optimized firing angles lead to a less ripple-afflicted direct current. The corresponding AC-line currents are shown in fig 6 and their space-phasor in fig. 7.

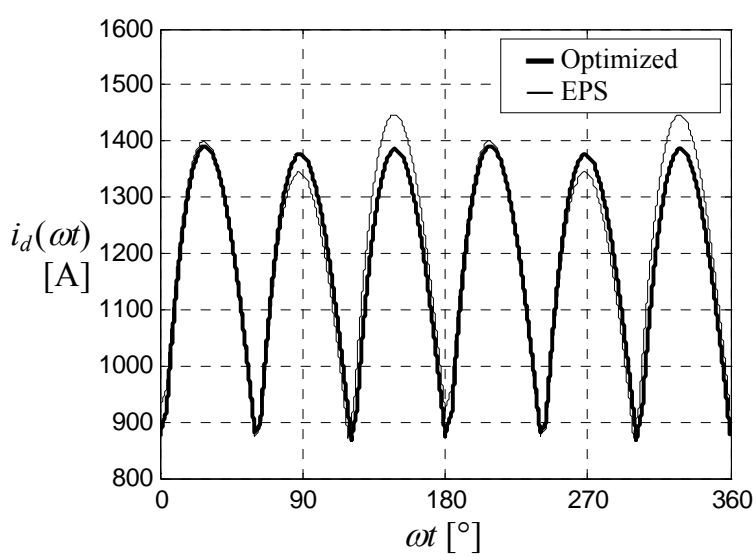

Fig. 5. Time-characteristic of the direct current

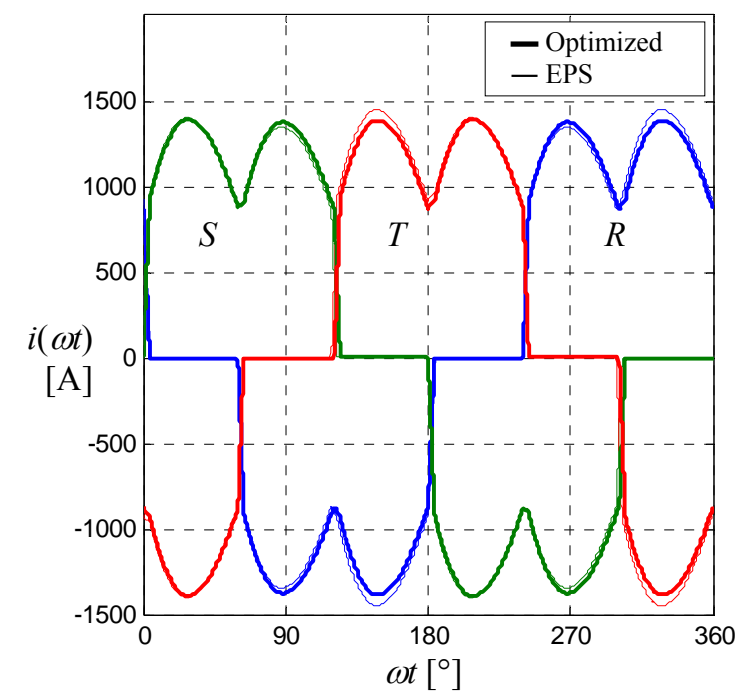

Fig. 6. Time-characteristics of the AC-line currents

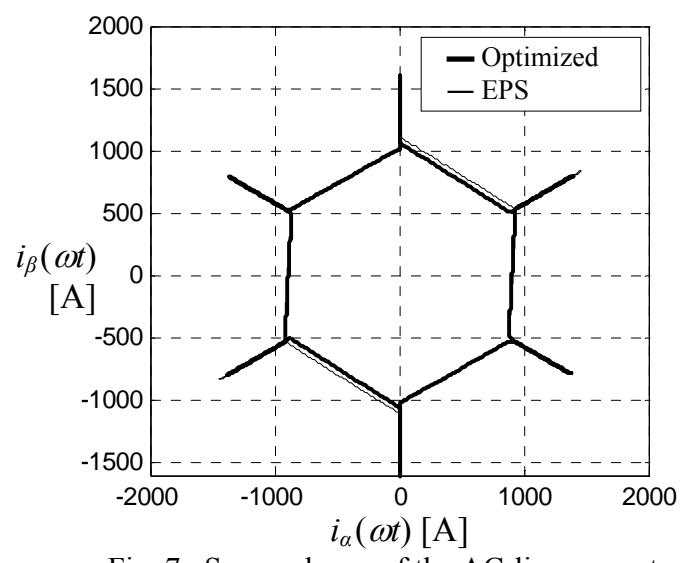

Fig. 7. Space-phasor of the AC-line currents

Fig. 6 and 7 lead to the same result: The optimized firing angles lead to a more balanced operation. This can also be seen in fig. 8 , which shows the frequency spectrum of the current space-phasor. The non-characteristic harmonics (e.g. the $-50 \mathrm{~Hz}$ and the $\pm 150 \mathrm{~Hz}$ harmonics) are damped by use of the optimization, while the characteristic harmonics nearly stay the same.

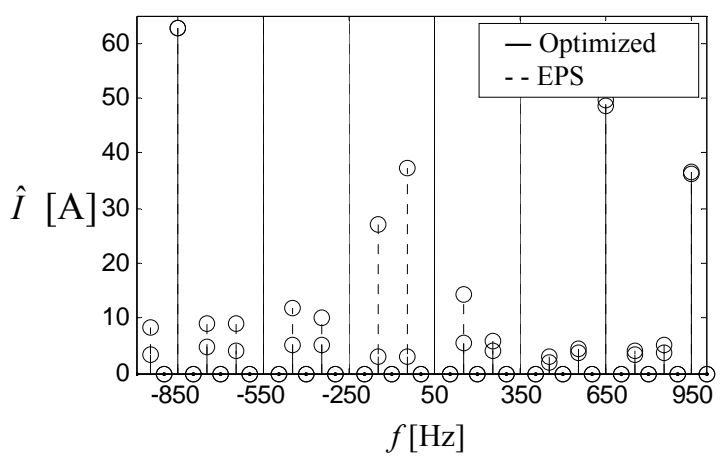

Fig. 8. Frequency spectrum of the current space-phasor

\section{Conclusion}

In this work, a method to calculate the operation of line-commutated current converters with unbalanced AC-line impedances is presented. A possible application is shown by means of an example, wherein a converters unbalanced operation is optimized. Though only small, improvements on a converter's behaviour in unsymmetrical operation can be achieved. Depending on the actual problem major improvements can be achieved.

\section{References}

[1] Sakui M., Fujita H.: Calculation of harmonic currents in a three-phase convertor with unbalanced power supply conditions, Proc. Inst. Elect. Eng., Vol. 139, Pt. B, No. 5, pp. 478-484, 1992

[2] Rashid M. H., Maswood A. I.: A novel method of harmonic assessment generated by three-phase AC-DC converters under unbalanced supply conditions, IEEE Trans. Ind. Applicat., Vol. 24, No. 4, pp. 590-597, 1988

[3] Yacamini R., Smith W. J.: Third-harmonic current from unbalanced AC/DC convertors, Proc. Inst. Elect. Eng., Vol. 130, Pt. C, No. 3, pp. 122-126, 1983

[4] Ngandui É., Olivier G., April G.-É., Guimaraes C.: DC harmonic distortion minimization of thyristor converters under unbalanced voltage supply using asymmetrical firing angle, IEEE Trans. Power Electron., Vol. 12, No. 2, pp. 332-342, 1997

[5] Hu J., He Y.: Modeling and control of grid-connected voltage-sourced converters under generalized unbalanced operation conditions, IEEE Trans. Energy Conv., Volume 23, Issue 3, pp. 903-913, 2008

[6] Saniter C., Wood A. R., Hanitsch R., Schulz, D.: Modelling the effects of AC system impedance unbalance on PWM converters using frequency coupling matrices, IEEE Power Tech Conference Proceedings, Vol. 2, Bologna, 2003

[7] Rendusara D., von Jouanne A., Enjeti P., Paice D. A.: Design considerations for six pulse and twelve pulse diode rectifier systems operating under voltage unbalance and pre-existing voltage distortion with some corrective measures, IEEE Ind. Applicat. Conf., pp. 2549-2556, 1995

[8] Weiland M., Först B., Herold G.: Investigation of six-pulse three-phase converters in unsymmetrical operation, 13th European Conf. on Power Electronics and Applicat. (EPE), Barcelona, 2009

[9] Weiland M., Herold G., Freudenstein T.: Investigation of line-commutated three-phase converters with impedance unbalance, 7th International Conference on Electric Power Quality and Supply Reliability PQ2010, Pt. D3, pp. 137-142, Kuressaare (Estonia), 2010 